\title{
BCLAF1 induces cisplatin resistance in lung cancer cells
}

\author{
TAO JIANG ${ }^{1 *}$, BINGJIE LIU $^{1 *}$, DONGPING WU ${ }^{2 *}$ and FENG ZHANG ${ }^{1}$ \\ ${ }^{1}$ College of Life Sciences, Shanghai Normal University, Shanghai 200234; ${ }^{2}$ Department of Radiation Oncology, \\ Shaoxing People's Hospital, Shaoxing Hospital of Zhejiang University, Shaoxing, Zhejiang 312000, P.R. China
}

Received January 20, 2020; Accepted July 6, 2020

DOI: $10.3892 / \mathrm{ol} .2020 .12090$

\begin{abstract}
Treatment for non-small cell lung cancer (NSCLC) remains challenging due to frequent recurrence and the development of resistance to platinum-based chemotherapy. The mechanism underlying NSCLC chemoresistance remains unclear. The present study aimed to investigate the mechanism of cisplatin resistance in NSCLC cells and it found that the expression of Bcl-2-associated transcription factor 1 (BCLAF1) was higher in the A549 cell line with cisplatin resistance (A549/DDP) by western blotting and reverse-transcription quantitative PCR, suggesting that elevated BCLAF1 expression is associated with acquired cisplatin resistance in A549 cells. BCLAF1 was found to promote DNA damage repair in A549/DDP cells by regulating $\gamma \mathrm{H} 2 \mathrm{~A}$ histone family member $\mathrm{X}$ foci formation by immunofluorescence and western blotting. BCLAF1 was also demonstrated to regulate ubiquitin-specific peptidase $22 \mathrm{mRNA}$ expression in A549/DDP cells, in addition to regulating $\mathrm{G}_{1}$ phase arrest by targeting p21 expression. Taken together, these findings suggest that BCLAF1 mediates cisplatin resistance by regulating the repair of DNA damage and p21-mediated $G_{1}$ phase arrest.
\end{abstract}

\section{Introduction}

Lung cancer is associated with one of the highest rates of mortality worldwide (1). Globally, lung cancer burden rose to 2.094 million new cases and 1.8 million cancer deaths in 2018 (2). Lung cancer is typically divided into two major subtypes: Small cell lung cancer and non-small cell lung

Correspondence to: Professor Feng Zhang, College of Life Sciences, Shanghai Normal University, 100 Guilin Road, Shanghai 200234, P.R. China

E-mail: fengz@shnu.edu.cn

Dr Dongping Wu, Department of Radiation Oncology, Shaoxing People's Hospital, Shaoxing Hospital of Zhejiang University, 568 Zhongxin Bei Road, Shaoxing, Zhejiang 312000, P.R. China E-mail: wudp666@163.com

*Contributed equally

Key words: non-small cell lung cancer, lung cancer, Bcl-2-associated transcription factor 1 , DNA damage, $G_{1}$ phase arrest, cisplatin resistance cancer (NSCLC) (3). NSCLC accounts for $\sim 80-85 \%$ of all lung cancer cases, where $>60 \%$ of patients with NSCLC present with locally advanced or advanced stages of the disease at the time of diagnosis (4). Platinum-based chemotherapy is currently the treatment of choice for patients with NSCLC (5). Cisplatin is a small molecule that is widely applied as a chemotherapeutic agent (6). Cisplatin binds to and crosslinks DNA, thereby disrupting DNA function, inhibiting mitosis and subsequently inducing apoptosis $(7,8)$. However, development of resistance to cisplatin has become a major obstacle for lung cancer treatment, the mechanism of which remains unknown, to the best of our knowledge. Accumulating evidence suggests that cisplatin resistance occurs following the dysregulation of gene transcription, which reduces the cellular accumulation of cisplatin, in turn inhibiting apoptosis, potentiating DNA repair and upregulating pro-survival signaling pathways to increase cell proliferation $(9,10)$. Therefore, understanding cisplatin resistance is necessary for the successful chemotherapeutic intervention of NSCLC.

Although a number of genes that are associated with the induction of resistance have previously been identified from differential gene expression profiles, identification of additional genes is required to illustrate the mechanism underlying cisplatin resistance. Bcl-2-associated transcription factor 1 (BCLAF1) was initially identified as a protein that interacts with the anti-apoptotic members of the Bcl-2 family (11). Subsequent studies have shown that BCLAF1 serves a key role in a wide range of physiological processes, including apoptosis, lung development, DNA repair and transcriptional regulation (12-14). Overexpression of BCLAF1 has previously been reported to induce apoptosis in a manner that could be reversed by expression of the anti-apoptotic protein Bcl-2 $(15,16)$. BCLAF1 has also been demonstrated to bind to BRCA1 in mediating resistance to DNA damage and formation of BRCA1-mRNA splicing complexes $(17,18)$. The documented multifaceted function of BCLAF1 in apoptosis, DNA repair and transcriptional regulation raises the possibility that BCLAF1 may also serve a crucial role in lung cancer cisplatin resistance. Therefore, in the present study, the potential effect of BCLAF on the induction of cisplatin resistance in NSCLC cells was explored.

\section{Materials and methods}

Cell lines. The human lung adenocarcinoma cell line A549/wild-type (WT) (The Cell Bank of Type Culture Collection of Chinese Academy of Sciences) and the 
cisplatin-resistant counterpart (A549/DDP), which were obtained by high-dose cisplatin shock ten times. When the fusion degree of A549 cells reached 70\%, $100 \mu \mathrm{M}$ cisplatin was added to the medium for $1 \mathrm{~h}$ and then these cells were cultured in fresh medium. Repeat this ten times. Cisplatin was purchased from Sigma-Aldrich; Merck KGaA. Both cell lines were maintained in DMEM (Sigma-Aldrich; Merck KGaA) supplemented with $10 \%$ fetal bovine serum (Gibco; Thermo Fisher Scientific, Inc.), $10 \mu \mathrm{l} / \mathrm{ml}$ penicillin-streptomycin solution (Hyclone; GE Healthcare Life Sciences) and cultivated at $37^{\circ} \mathrm{C}$ in $5 \% \mathrm{CO}_{2}$. In addition, the A549/DDP cell medium contained $4 \mu \mathrm{M}$ cisplatin to maintain the drug-resistant phenotype. All cells used for the experiments were in logarithmic phases of growth.

Transfection of small interferring RNA (siRNA) and plasmid. To inhibit the expression of BCLAF1 and USP22, negative control siRNA, USP22-siRNA and BCLAF1-siRNA were purchased from Shanghai GenePharma Co., Ltd. The same negative control siRNA was used for both BCLAF1 and USP22 siRNA transfections. The sequences were as follows: Negative control siRNA, 5'-UUACAGAGTAACTUCUUA UC-3'; BCLAF1-siRNA-1, 5'-UCACAUUCU UCA AGG UCAATT-3'; and BCLAF1-siRNA-2, 5'-CCGGUCAUAUAG AUCUUCUTT-3'. The sequences of USP22-siRNA-1 and USP22-siRNA-2 were as follows: 5'-CACGGACAGUCUCAA CAAUTT-3' and 5'-GGAGAAAGAUCACCUCGAATT-3'. In total, 200 pmol BCLAF1-siRNA or ubiquitin-specific peptidase 22 (USP22)-siRNA was transfected into A549/WT and A549/DDP cells using Lipofectamine ${ }^{\circledR} 2000$ (Thermo Fisher Scientific, Inc.) according to manufacturer's protocol. 200 pmol negative control siRNA was also transfected into A549/WT cells and A549/DDP using Lipofectamine ${ }^{\circledR} 2000$ (Thermo Fisher Scientific, Inc.). For overexpression of BCLAF1, full length CDNA of BCLAF1 was cloned into the pS-Flag-SBP vector (Addgene, Inc.). An empty vector was used as the negative control. pS-Flag-SBP vector was obtained as previously described (19). A solution containing $5 \mu \mathrm{g}$ plasmid and $10 \mu \mathrm{l} \mathrm{ViaFect}{ }^{\mathrm{TM}}$ Transfection Reagent (Promega Corporation) was transfected into A549 or A549/DDP cells. Follow up experiments were performed $24 \mathrm{~h}$ after transfection.

Cell viability assay. The in vitro chemosensitivity of cells was analyzed using MTT assay. Briefly, cells were seeded into sterile 96-well plates at a density of $5 \times 10^{3}$ cells/well followed by attachment overnight. The cells were then treated with different concentrations $(6.25,12.5,25,50$ and $100 \mu \mathrm{M})$ of cisplatin for $36 \mathrm{~h}$ at room temperature, following which $10 \mu \mathrm{l}$ MTT was added to each well. Following $4 \mathrm{~h}$ MTT incubation, $10 \%$ SDS was added into each well and the cells were cultivated at $37^{\circ} \mathrm{C}$ in $5 \% \mathrm{CO}_{2}$ overnight. A spectrophotometer was then used to measure absorbance in each well at $570 \mathrm{~nm}$. MTT experiments were performed at least three times.

Colony formation assays. A549/WT and A549/DDP cells transfected with NC or BCLAF1 siRNA were plated in 6-well culture dishes at a density of $5 \times 10^{4} \mathrm{cells} / \mathrm{ml}$ and exposed to $40 \mu \mathrm{M}$ cisplatin continuously for $36 \mathrm{~h}$ at room temperature. The cells were fixed with methanol for $15 \mathrm{~min}$ at roon temperature and stained with $1 \%$ crystal violet for $15 \mathrm{~min}$ at room temperature. Cells were observed with a fluorescence microscope (Olympus-BX53; Olympus Corporation; magnification, $\mathrm{x} 40$ ).

Reverse transcription-quantitative PCR (RT-qPCR). For RNA extraction and RT-qPCR, total RNA was isolated from A549/WT and A549/DDP cultured cells using TRIzol ${ }^{\circledR}$ reagent (Invitrogen; Thermo Fisher Scientific, Inc.). cDNA was synthsized from total RNA using M-MLV Reverse Transcriptase (Promega Corporation) according to the manufacturer's protocols. Subsequent qPCR reactions were then performed in a volume of $10 \mu \mathrm{l}$ that included $1 \mu \mathrm{l} \mathrm{cDNA}$ template, $0.8 \mu \mathrm{l}$ $10 \mu \mathrm{M}$ primers (contains forward and reverse primers), $0.8 \mu \mathrm{l}$ 50X ROX Reference Dye (used to calibrate the instrument), $5 \mu 12 \mathrm{X}$ TB Green ${ }^{\circledR}$ Premix Ex Taq ${ }^{\mathrm{TM}}$ II (Tli RNaseH Plus;

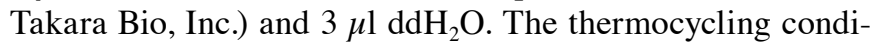
tions were $95^{\circ} \mathrm{C}$ for $5 \mathrm{sec}$ and $60^{\circ} \mathrm{C}$ for $30 \mathrm{sec}$ for 45 cycles. The sequences of primers used were as follows: Homo-GAPDH forward, 5'-GCACCGTCAAGGCTGAGAAC-3' and reverse, 5'-TGGTGA AGACGCCAGTGGA-3'; USP22 forward, 5'-GGAAAATGCAAGGCGTTGGAGA-3' and reverse, 5'-GTGCAGTTCGAGGTGATCTTT-3'; p21 forward, 5'-CAT GCCAGCTACTTCCTCCT-3' and reverse, 5'-CAGGTCTGA GTGTCCAGGAA-3'; and BCLAF1 forward, 5'-TCTGGA ATAGAAGGCACTCTAGG-3' and reverse, 5'-ACCCTCGTC TTTTAGAAACAGGA-3'. The instrument used was CFX96 ${ }^{\mathrm{TM}}$ Real-Time PCR Detection system (Bio-Rad Laboratories, Inc.). The relative mRNA expression was quantified using the $2^{-\triangle \Delta C q}$ method (20), where the expression level of GAPDH mRNA was used as an internal control for normalization. All experiments were performed in duplicate, each being repeated a minimum of three times.

Immunofluorescence. A549/WT and A549/DDP cells treated with $4 \mu \mathrm{M}$ cisplatin were incubated at $37^{\circ} \mathrm{C}$ in $5 \% \mathrm{CO}_{2}$ for $24 \mathrm{~h}$, before being fixed with $3 \%$ paraformaldehyde for $20 \mathrm{~min}$ at room temperature. The cells were then incubated with $0.5 \%$ Triton X-100 for $10 \mathrm{~min}$ at room temperature, following which they were blocked with $10 \%$ goat serum (Sangon Biotech) for $1 \mathrm{~h}$ at room temperature before incubation with the anti- $\gamma \mathrm{H} 2 \mathrm{~A}$ histone family member $\mathrm{X}(\gamma \mathrm{H} 2 \mathrm{AX})$ antibody (1:1,000; cat. no. D7T2V; Cell Signaling Technology, Inc.) or the anti-BCLAF1 (1:1,000; cat. no. A300-608A; Bethyl Laboratories) antibody dissolved in PBS containing $5 \%$ goat serum at $4^{\circ} \mathrm{C}$ overnight. The next day, the cells were stained with the secondary antibodies FITC AffiniPure goat anti-rabbit IgG (H+L) (1:200; cat. no. 111-095-144; Jackson Immunoresearch) and AffiniPure goat anti-mouse $\operatorname{IgG}(\mathrm{H}+\mathrm{L})$ (1:200; cat. no. 115-025-146; Jackson Immunoresearch) dissolved in PBS containing 5\% goat serum at room temperature for $1 \mathrm{~h}$. Nuclei were then stained using DAPI (Invitrogen; Thermo Fisher Scientific, Inc.) for $5 \mathrm{~min}$ at room temperature. A fluorescence microscope was used (magnification, x600). Quantitative image analysis was performed using Image $\mathbf{J}$ version 1.8.0 (National Institutes of Health).

Western blot analysis. Total proteins of A549 or A549/DDP cells were extracted using an effective lysing fraction of $1 \%$ Triton X-100 dissolved in RIPA lysis buffer (EpiZyme Biotech). An appropriate amount of lysate was incubated with a protease 
A

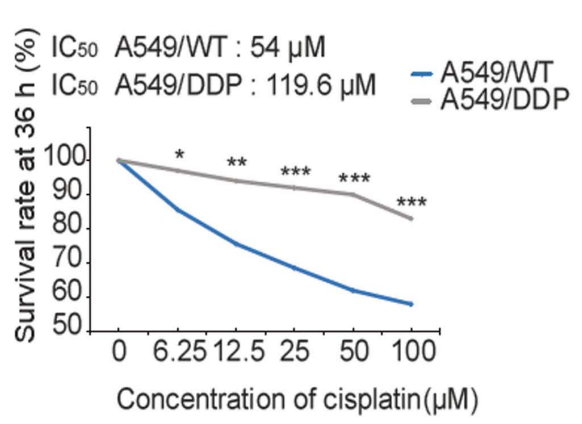

C

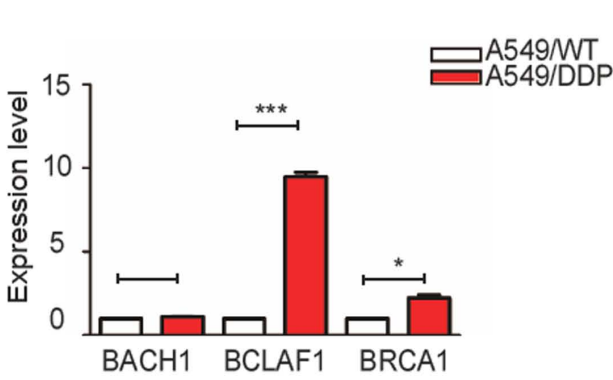

E

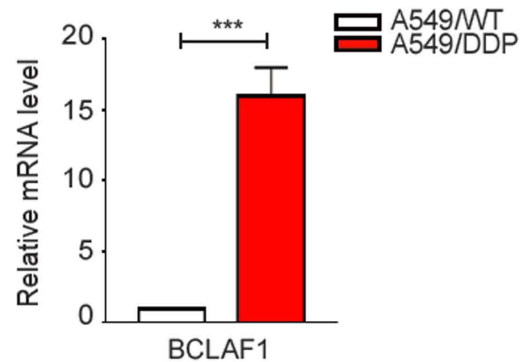

B

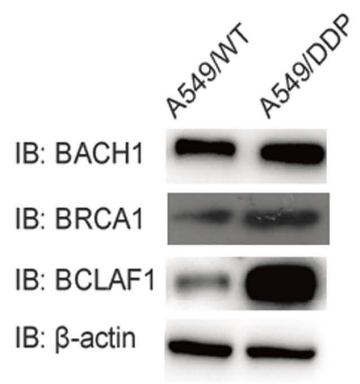

D

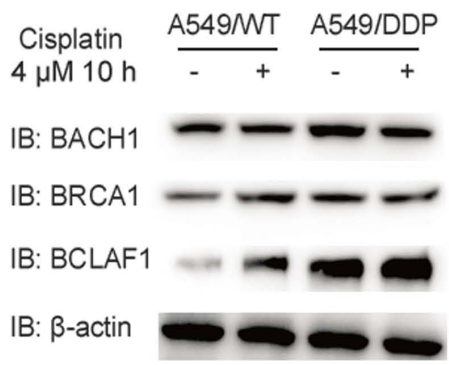

Figure 1. BCLAF1 expression is upregulated in A549/DDP cells. (A) MTT assays were used to measure the sensitivity of A549/WT and A549/DDP cells to cisplatin. Cells were treated with different concentrations of cisplatin for $36 \mathrm{~h} .{ }^{*} \mathrm{P}<0.05,{ }^{* *} \mathrm{P}<0.01,{ }^{* * *} \mathrm{P}<0.001$ vs. A549/WT. (B) BRCA1, BACH1 and BCLAF1 protein expression levels were measured in A549/WT and A549/DDP cells by western blotting and $(\mathrm{C})$ were quantified. ${ }^{*} \mathrm{P}<0.05$, ${ }^{* * *} \mathrm{P}<0.001$. (D) A549/WT and A549/DDP cells were treated continuously with either 0 or $4 \mu \mathrm{M}$ cisplatin for $10 \mathrm{~h}$, following which BRCA1, BACH1 and BCLAF1 protein expression were examined by western blotting. (E) BCLAF1 mRNA expression was measured using reverse transcription-quantitative PCR in A549/WT and A549/DDP cells. Data are presented as the means \pm SD from three independent experimental repeats. ${ }^{*} \mathrm{P}<0.05,{ }^{* * *} \mathrm{P}<0.001$. DDP, cisplatin resistance; WT, wild-type; BCLAF1, bcl-2-associated transcription factor 1; BACH1, BTB domain and CNC homolog 1.

inhibitor (1:100) for $1 \mathrm{~min}$ before use. The protein concentration was determined using a bicinchonininc acid (BCA) assay (Thermo Fisher Scientific Inc.). The protein samples $(20 \mu \mathrm{g}$ of protein loaded per lane) were separated by 7.5 or $15 \%$; SDS-PAGE (90V, $90 \mathrm{~min})$ and transferred (70V, $90 \mathrm{~min})$ onto PVDF membranes (Immobilon-P; EMD Millipore). Membranes were blocked with a TBS buffer $(50 \mathrm{mM}$ Tris- $\mathrm{HCl}$, $150 \mathrm{mM} \mathrm{NaCl}, \mathrm{pH}$ 7.4) supplemented with 5\% skimmed milk and $0.1 \%$ Tween-20 for $30 \mathrm{~min}$ at room temperature, followed by incubation with primary antibodies against BCLAF1 (1:2,500; cat. no. A300-608A; Bethyl Laboratories), p21 (1:2,500; cat. no. ab109520; Abcam), BTB domain and CNC homolog 1 (BACH1; 1:2,500; cat. no. 4578s; Cell Signaling Technology, Inc.), cyclin D1 (1:2,500; cat. no. 55506; Cell Signaling Technology, Inc.), BRCA1 (1:2,500; cat. no. 14823; Cell Signaling Technology, Inc.), $\gamma \mathrm{H} 2 \mathrm{AX}(1: 2,500$; cat. no. D7T2V; Cell Signaling Technology, Inc.), USP22 (1:2,500; cat. no. EPR18945; Abcam) or $\beta$-actin $(1: 2,500$; cat. no. IPSC1030, Sigma-Aldrich, Merck KgaA) at $4^{\circ} \mathrm{C}$ overnight. Membranes were incubated with the secondary antibodies goat anti-mouse IgG (H+L)-HRP (1:5,000; cat. no. PB001; CASICO) and mouse anti-rabbit $\mathrm{IgG}$, light chain specific (1:5,000; cat. no. 211-032-171; Jackson Immunoresearch) at room temperature for $1 \mathrm{~h}$. For visualization, membranes were incubated with the Light Chemiluminescence (Omni-ECL ${ }^{\mathrm{TM}}$ Femto Light Chemiluminescence kit; cat. no. SQ201; EpiZyme Biotech). Western blot results were analyzed by Image $\mathbf{J}$ version 1.8.0 (National Institutes of Health).

Cell cycle analysis. A549/DDP cells transfected with either BCLAF1 siRNA or negative control siRNA were used for cell cycle analysis. Cell cycle progression was determined by propidium iodide (PI) staining using a flow cytometer. Briefly, cells were fixed with $70 \%$ cold ethanol at $4^{\circ} \mathrm{C}$ overnight, washed twice with ice-cold PBS, and incubated with $10 \mathrm{mg} / \mathrm{ml}$ RNase at $37^{\circ} \mathrm{C}$. Cell cycle was monitored by PI staining of nuclei for 


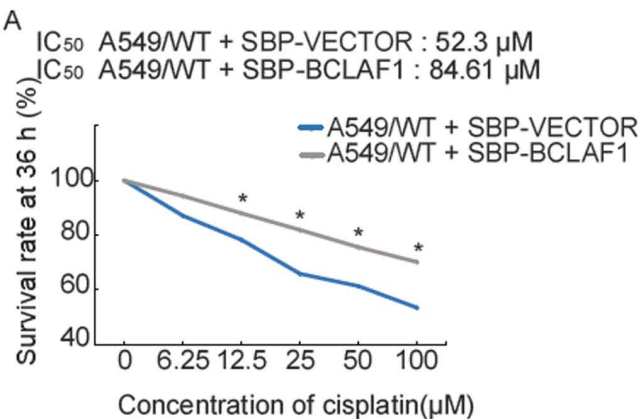

B

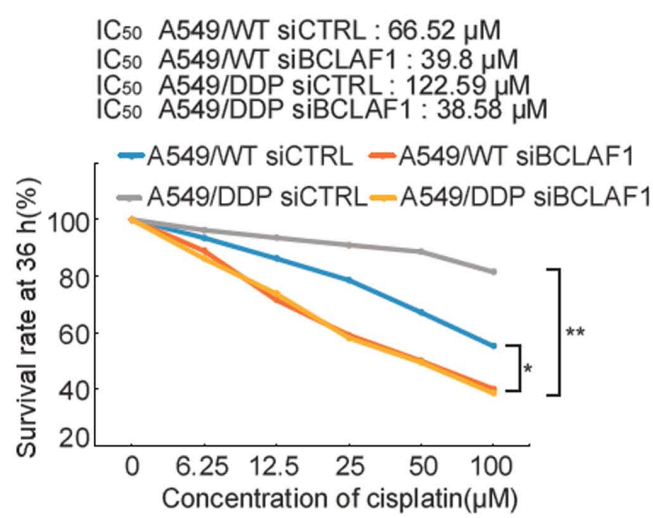

C Cisplatin
$40 \mu \mathrm{M} 36 \mathrm{~h}$
$\mathrm{~A} 549 \mathrm{~N}$ A549NT SICTRL A549NT SIBCLAF1
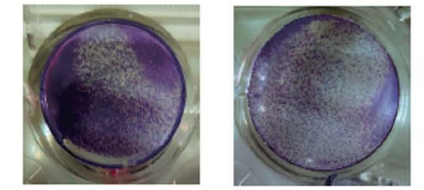

A549/DDP siCTRL A549/DDP siBCLAF1

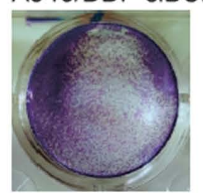

D

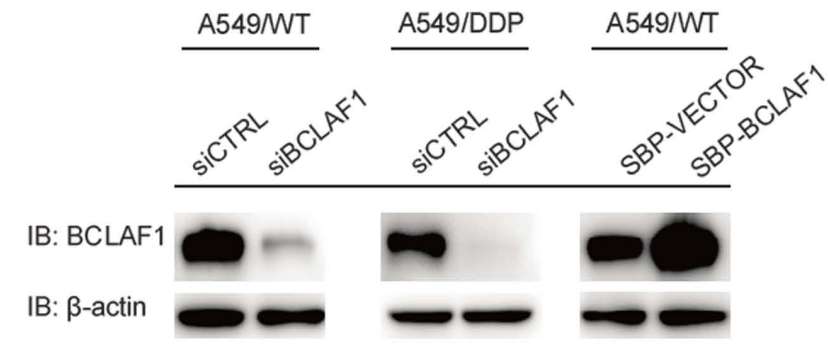

Figure 2. Elevated BCLAF1 expression levels are associated with acquired cisplatin resistance in A549 cells. (A) MTT assay was used to measure the sensitivity to cisplatin in A549/WT cells transfected with either the SBP-vector or SBP-BCLAF1. Cells stably expressing the SBP-vector or SBP-BCLAF1 were treated with different concentrations of cisplatin for $36 \mathrm{~h}$. ${ }^{*} \mathrm{P}<0.05 \mathrm{vs}$. A549/WT + SBP-vector. (B) MTT assays were used to measure the sensitivity to cisplatin in A549/WT cells transfected with siCTRL or siBCLAF1 and in A549/DDP cells transfected with siCTRL or siBCLAF1, following which they were treated with different concentrations of cisplatin for $36 \mathrm{~h}$. ${ }^{*} \mathrm{P}<0.05,{ }^{* *} \mathrm{P}<0.01$. (C) The colony-forming ability of A549/WT cells transfected with siCTRL or siBCLAF1 and in A549/DDP cells transfected with siCTRL or siBCLAF1 to cisplatin was examined after exposure to $40 \mu \mathrm{M}$ cisplatin continuously for $36 \mathrm{~h}$. (D) BCLAF1 protein expression were measured by western blotting in A549/WT cells transfected with siCTRL, siBCLAF1, SBP-vector or SBP-BRCAF1 and in A549/DDP cells transfected with siCTRL or siBCLAF1. Data are presented as the means \pm SD from three independent experimental repeats. SBP, streptavidin-binding peptide; DDP, cisplatin resistance; WT, wild-type; BCLAF1, bcl-2-associated transcription factor 1; si, small interfering; CTRL, control.

30 min at room temperature, and PI uptake was analyzed by fluorescence-activated cell sorting using a flow cytometer $\left(\mathrm{CyAn}^{\mathrm{TM}}\right.$ ADP Analyzer; Beckman Coulter, Inc.). Results were analyzed by Modfit LT version 5.0 (Verity Software House, Inc.).

Statistical analysis. All statistical analyses were performed using GraphPad Prism software (version 7; GraphPad Software, Inc.). Data are presented as the mean \pm standard devaition from at least three independent experiments. Statistical significance was determined by one-way analysis of variance followed by Bonferroni's test. Comparisons between two group were performed by unpaired Student's t-test. $\mathrm{P}<0.05$ was considered to indicate a significantly significant difference.

\section{Results}

BCLAF1 expression is upregulated in the cisplatin-resistant A549/DDP cell line. To study the chemoresistance of NSCLC, cisplatin-resistant NSCLC cell line models (A549/DDP) were first established by treating the A549 cell line with cisplatin for 2 months. Surviving cells after this period were then tested for cisplatin sensitivity using a MTT assay. Results from the MTT assay demonstrated that A549/DDP cells exhibited a significantly increased cisplatin resistance compared with that of the parental A549 cell line (Fig. 1A), and the $\mathrm{IC}_{50}$ value of cisplatin for A549/DDP was 1.2-fold higher compared with that for A549/WT $(119.6 \mu \mathrm{M}$ vs. $54 \mu \mathrm{M})$. 


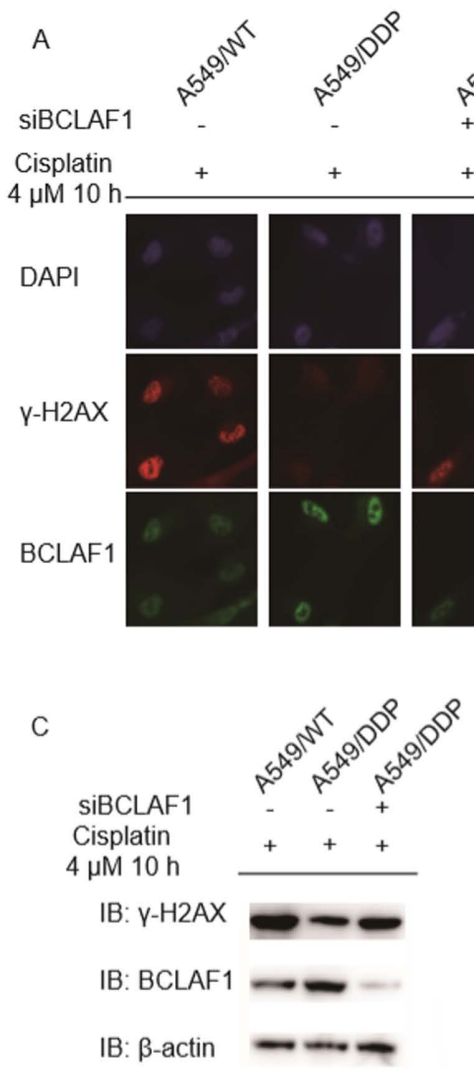

$\mathrm{E}$

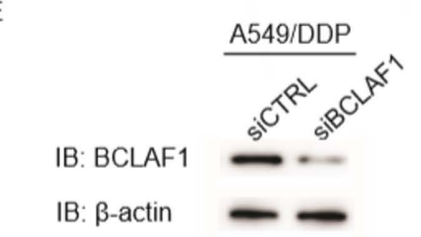

B
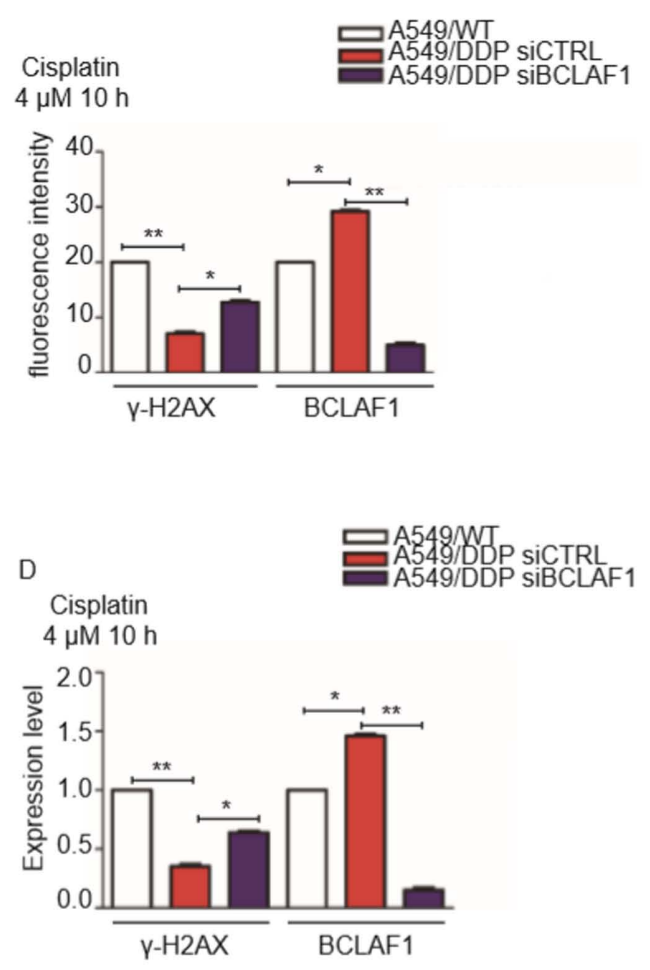

Figure 3. DNA damage repair in A549/DDP cells is regulated by BCLAF1. (A) Representative immunofluorescence images of $\gamma \mathrm{H} 2 \mathrm{AX}$ and BCLAF1 in A549/WT and A549/DDP cells transfected with siBCLAF1 or siCTRL. Cell lines were treated with $4 \mu \mathrm{M}$ cisplatin for $10 \mathrm{~h}$. Magnification, $\mathrm{x} 600$. (B) Quantification of $\gamma \mathrm{H} 2 \mathrm{AX}$ and BCLAF1 fluorescence intensity in A549/WT and A549/DDP cells transfected with siBCLAF1 or siCTRL. (C) The protein expression levels of $\gamma \mathrm{H} 2 \mathrm{AX}$ and BCLAF1 were measured by western blotting in A549/WT and A549/DDP cells transfected with siBCLAF1 or siCTRL. Cells were treated with $4 \mu \mathrm{M}$ cisplatin for $10 \mathrm{~h}$. (D) Quantification of $\gamma \mathrm{H} 2 \mathrm{AX}$ and BCLAF1 protein expression in A549/WT and A549/DDP cells transfected with siBCLAF1 or siCTRL. (E) The expression levels of BCLAF1 was measured in A549/DDP cells by western blotting. Cells were transfected with either control or BCLAF1 siRNA for $48 \mathrm{~h}$. Data are presented as the means \pm SD from three independent experimental repeats. ${ }^{*} \mathrm{P}<0.05,{ }^{* *} \mathrm{P}<0.01$. DDP, cisplatin resistance; WT, wild-type; BCLAF1, bcl-2-associated transcription factor 1; si, small interfering; CTRL, control; $\gamma \mathrm{H} 2 \mathrm{AX}, \gamma \mathrm{H} 2 \mathrm{~A}$ histone family member X.

It has previously been reported that increased tolerance to DNA damage is one of the major mechanisms of cisplatin resistance $(10,21,22)$. Therefore, the expression levels of several key components of the DNA damage response, including BRCA1, BACH1 and BCLAF1, were examined in A549/DDP cells. No change in the expression level of BACH1 was observed in A549/DDP cells compared with in A549 cells, whilst both BRCA1 and BACH1 protein expression was increased in A549/DDP cells compared with A549/WT cells (Fig. 1B-D). When compared with A549/WT cells, in A549/DDP cells the protein expression level of BCLAF1 is significantly higher than that of BRCA1, hence the functional link between BCLAF1 and cisplatin resistance in NSCLC was further explored. When BCLAF1 levels were investigated further it was found that BCLAF1 mRNA level also significantly higher in the cisplatin-resistant A549/DDP cells compared with A549/WT cells (Fig. 1E).
Elevated BCLAF1 expression levels are associated with acquired cisplatin resistance in A549 cells. BCLAF1 is a multifunctional protein that has been implicated in a number of cellular functions, including apoptosis (16), lung development and transcriptional regulation (14,23). As aforementioned, the present study demonstrated that BCLAF1 expression levels were significantly higher in A549/DDP cells compared with in A549 cells, prompting further examination into whether BCLAF1 exerts an effect on cisplatin resistance in A549/ DDP cells. Therefore, the association between BCLAF1 expression and cisplatin sensitivity in NSCLC cell lines was next evaluated by first testing the effects of BCALF1 overexpression on cisplatin resistance in A549 cells. A549 cells transfected with the BCLAF1 overexpression vector or the empty vector were treated with cisplatin, following which cisplatin sensitivity was determined using MTT assay. The $\mathrm{IC}_{50}$ for cisplatin in A549 cells transfected with the empty vector was calculated to 


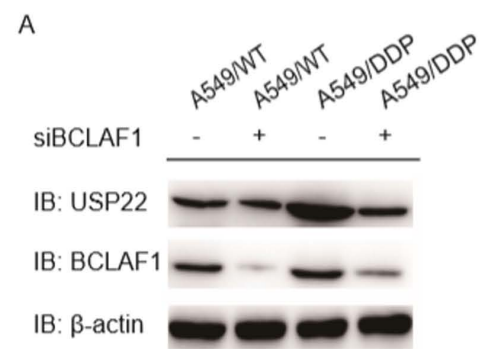

B
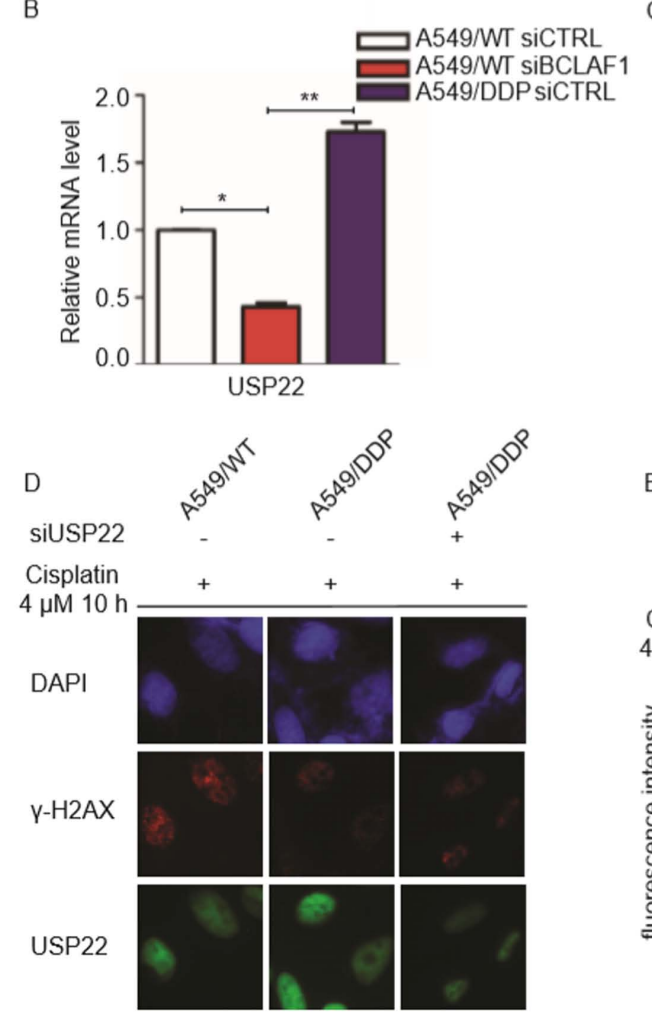

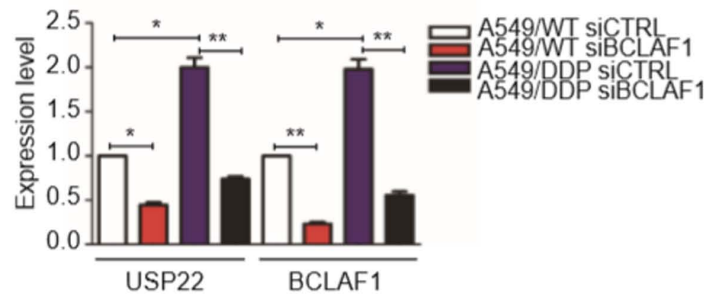

C

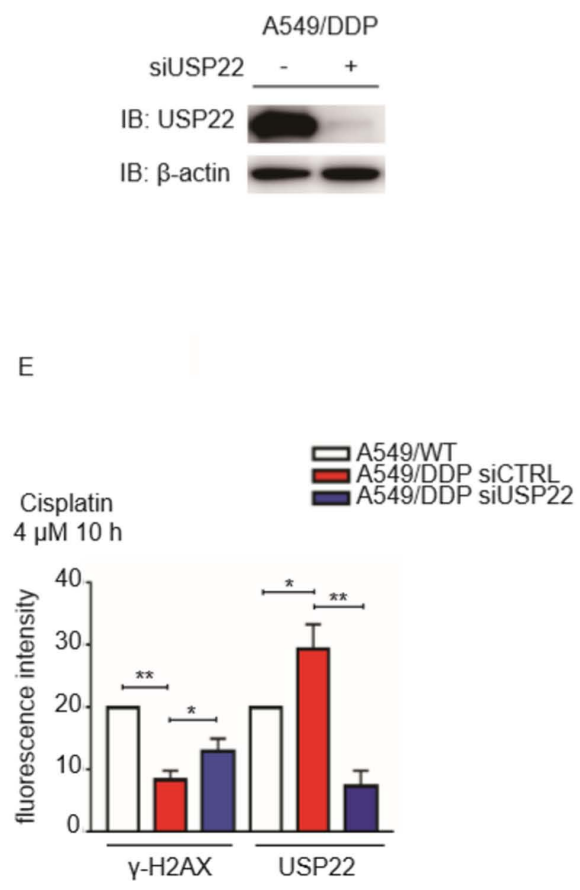

Figure 4. Expression of BCLAF1 is associated with USP22 expression in A549/DDP cells. (A) The expression levels of USP22 and BCLAF1 were measured and quantified in A549/WT and A549/DDP cells by western blotting. Cells were transfected with either control or BCLAF1 siRNA for 48 h. (B) USP22 mRNA expression was measured using reverse transcription-quantitative PCR in A549/WT cells transfected with siCTRL or siBCLAF1 and A549/DDP cells transfected with siCTRL. (C) The expression level of USP22 was measured in A549/DDP cells by western blotting. Cells were transfected with either control or USP22 siRNA for $48 \mathrm{~h}$. (D) Representative immunofluorescence images of $\gamma \mathrm{H} 2 \mathrm{AX}$ and USP22 in A549/WT and A549/DDP cells transfected with siUSP22 or siCTRL. Cells were treated with $4 \mu \mathrm{M}$ cisplatin for $10 \mathrm{~h}$. Magnification, $\mathrm{x} 600$. (E) Quantification of $\gamma \mathrm{H} 2 \mathrm{AX}$ and USP22 fluorescence intensity in A549/WT and A549/DDP cells transfected with siUSP22 or siCTRL. Data are presented as the means \pm SD from three independent experimental repeats. ${ }^{*} \mathrm{P}<0.05$, ${ }^{* *} \mathrm{P}<0.01$. DDP, cisplatin resistance; WT, wild-type; BCLAF1, bcl-2-associated transcription factor 1; si, small interfering; CTRL, control; $\gamma \mathrm{H} 2 \mathrm{AX}, \gamma \mathrm{H} 2 \mathrm{~A}$ histone family member X; USP22, ubiquitin-specific peptidase 22.

be $52.3 \mu M$. By contrast, following overexpression of BCLAF1 the $\mathrm{IC}_{50}$ was $84.61 \mu M$, and cell viability was found to be significantly increased (Fig. 2A). These observations suggest that the upregulation of BCLAF1 can promote cisplatin resistance in A549 cells. MTT assays subsequently demonstrated that knockdown of BCLAF1 expression using siRNA reduced the $\mathrm{IC}_{50}$ of A549/WT cells from 66.52 to $39.9 \mu \mathrm{M}$, and cell viability was significantly reduced (Fig. 2B), supporting the notion further that the depletion of BCLAF1 can reverse the cisplatin resistance of A549/DDP cells. Data from the colony formation assays following cisplatin treatment showed that BCLAF1-knockdown significantly reduced A549 cell viability (Fig. 2C and D), proving that BCLAF1 can promote A549 cell viability. Furthermore, in A549/DDP cells, knockdown of BCLAF1 expression reduced the $\mathrm{IC}_{50}$ for cisplatin from 122.59 to $38.58 \mu \mathrm{M}$, and significantly inhibited cell viability (Fig. 2B). These results suggested that BCLAF1 is a key component in mediating cisplatin sensitivity in A549/DDP cells.

DNA damage repair in A549/DDP cells is under the regulation of $B C L A F 1$. It has previously been reported that BCLAF1 is involved in the $\gamma \mathrm{H} 2 \mathrm{AX}$-mediated regulation of apoptosis and DNA repair (16). Therefore, the effects of BCLAF1 on DNA damage repair and $\gamma \mathrm{H} 2 \mathrm{AX}$ foci formation were examined in A549/DDP cells. Since phosphorylation of $\gamma \mathrm{H} 2 \mathrm{AX}$ at the ser-139 residue is a sensitive early genotoxic biomarker in cisplatin-induced DNA double strand breaks (DSBs) $(24,25)$, the relationship between $\gamma \mathrm{H} 2 \mathrm{AX}$ foci formation and the levels of BCLAF1 expression were examined further to explore the effects of BCLAF1 on cisplatin-induced DNA damage repair. 
A

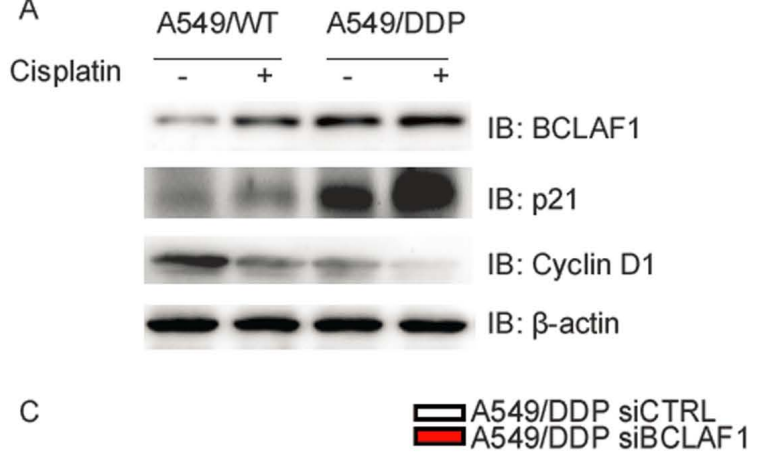

B

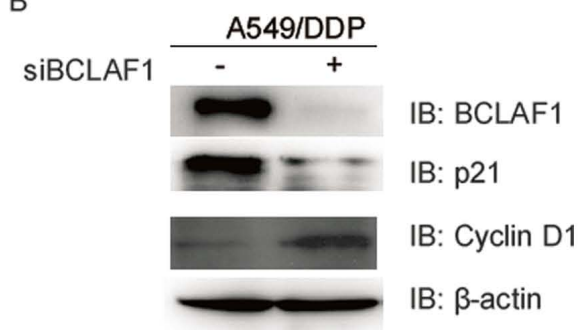

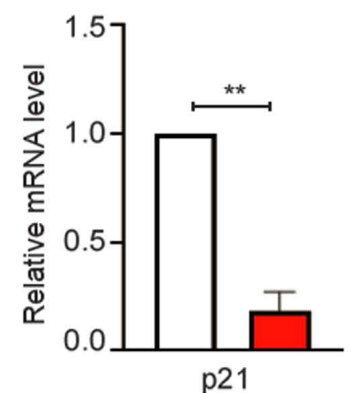

D
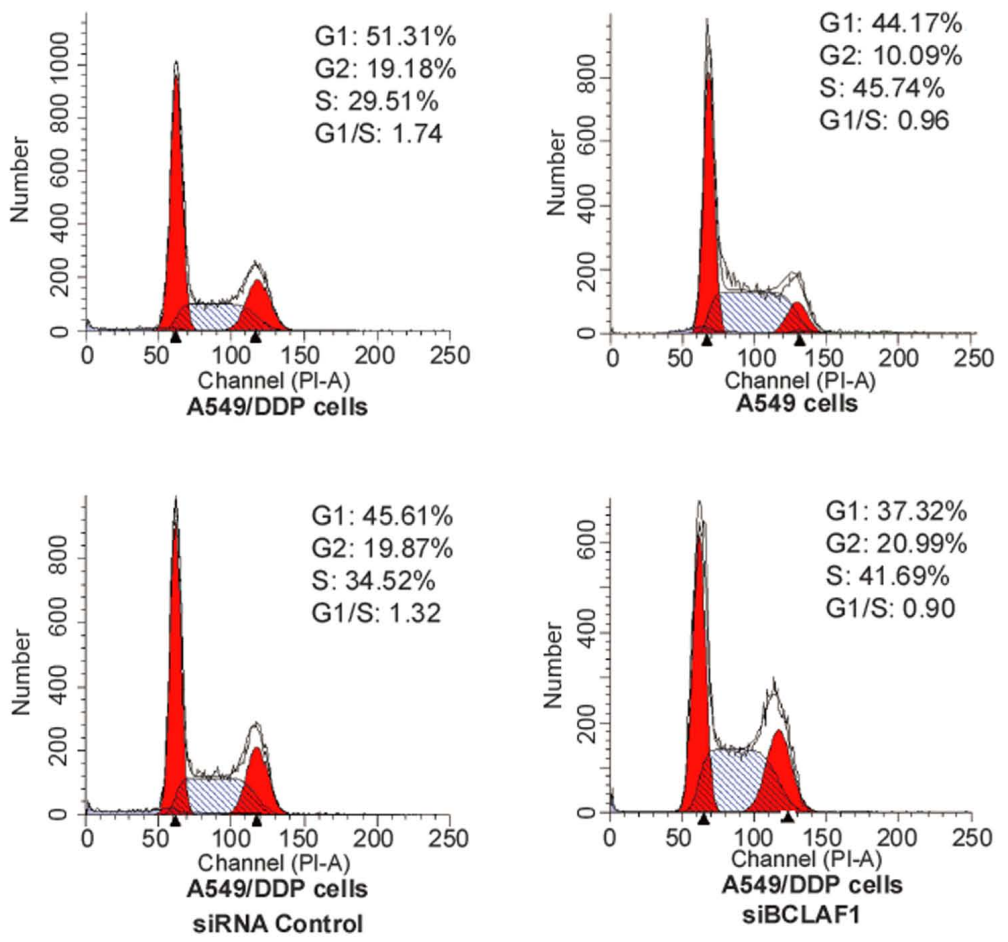

Figure 5. BCLAF1 regulates $\mathrm{G}_{1}$ phase arrest by targeting p21 expression. (A) The expression levels of BCLAF1, p21 and Cyclin D1 were measured in A549/WT and A549/DDP cells by western blotting. Cells were treated with or without $4 \mu \mathrm{M}$ cisplatin. (B) The expression levels of BCLAF1, p21 and Cyclin D1 were measured in A549/DDP and A549/DDP cells transfected with siBCLAF1 or siCTRL were measured by western blotting. (C) p21 mRNA expression was measured using reverse transcription-quantitative PCR in A549/DDP cells transfected with either siCTRL or siBCLAF1. (D) Cell cycle analysis of A549, A549/DDP and A549/DDP. Cells were transfected with either siBCLAF1 or siCTRL. Data are presented as the means \pm SD from three independent experimental repeats ${ }^{* *} \mathrm{P}<0.01$. DDP, cisplatin resistance; WT, wild-type; BCLAF1, bcl-2-associated transcription factor 1; si, small interfering; CTRL, control.

Following cisplatin treatment, the number of $\gamma \mathrm{H} 2 \mathrm{AX}$ foci in A549/DDP cells was found to be significantly lower compared with that in A549/WT cells, though the intensity of nuclear BCLAF1 staining was observed to be significantly higher in A549/DDP cells (Fig. 3A-B). This suggests that elevated BCLAF1 expression in A549/DDP cells enhances DSB repair and thus induces cisplatin resistance. Next, to verify the association between BCLAF1 expression and DNA damage repair following treatment with cisplatin, $\gamma \mathrm{H} 2 \mathrm{AX}$ foci formation were examined in A549/DDP cells following the depletion of BCLAF1 expression (Fig. 3A and B). The results of western blotting further verified the aforementioned result (Fig. $3 \mathrm{C}$ and D). The results demonstrated that BCLAF1-knockdown in A549/DDP cells significantly increased the number of cisplatin-induced $\gamma \mathrm{H} 2 \mathrm{AX}$ foci, suggesting that BCLAF1 
induces cisplatin resistance by facilitating DSB repair in A549/DDP cells.

Expression of BCLAF1 associates with that of USP22 in $A 549 / D D P$ cells. It has been reported previously that USP22 overexpression can mediate cisplatin resistance in A549 cells, where it modulates Ku70/BAX-dependent apoptosis and $\gamma \mathrm{H} 2 \mathrm{AX}$-mediated DNA damage repair (26). Since BCLAF1 overexpression was also observed to enhance cisplatin resistance in A549 cells, the association between the expression levels of BCLAF1 and USP22 was next analyzed in A549/DDP cells. The results demonstrated that the protein expression levels of USP22 and BCLAF1 were significantly higher in A549/DDP cells compared with those in A549/WT cells (Fig. 4A and B). Notably, following knockdown of BCLAF1 expression, USP22 protein expression was also found to be significantly reduced in A549/DDP cells (Fig. 4A). These results indicated a positive association between BCLAF1 and USP22 expression. As BCLAF1 can also function as a transcriptional factor to regulate target gene expression (27), the hypothesis that BCLAF1 can directly regulate USP22 expression was considered. Supporting this, USP22 mRNA expression was found to be significantly reduced following BCLAF1 downregulation in A549/WT cells. By contrast, USP22 mRNA expression was observed to be markedly elevated in A549/DDP cells with higher expression of BCLAF1 (Fig. 4A and B), suggesting that BCLAF1 regulates USP22 expression at the mRNA level. Following USP22 knockdown in A549/DDP (Fig. 4C), it was subsequently found that USP22-knockdown in A549/DDP cells significantly increased the number of cisplatin-induced $\gamma \mathrm{H} 2 \mathrm{AX}$ foci (Fig. 4D and E), suggesting that BCLAF1 can modulate USP22 expression to facilitate DNA damage repair.

BCLAF1 regulates $G_{1}$ phase cell cycle arrest by targeting $p 21$ expression. It has been shown that BCLAF1 co-ordinates with BRCA1 as a component of the RNA splicing complex, which regulates the stability of cyclin D1 and BRCA1 mRNA (17). Since BCLAF1 expression was found to be increased in A549/DDP cells, the expression levels of cyclin D1 and p21 in A549/WT and A549/DDP cells with or without cisplatin treatment were compared. Following cisplatin treatment, the expression levels of BCLAF1 and p21 were revealed to be increased in A549/DDP cells, whilst cyclin D1 expression were demonstrated to be reduced (Fig. 5A). Depletion of BCLAF1 expression in A549/DDP cells lead to a reduction in p21 expression but increase in cyclin D1 expression (Fig. 5B. These findings suggest that BCLAF1 serves an important role in the regulation of p21 and cyclin D1 gene expression.

Elevated p21 protein levels have previously been documented to inhibit cyclin D-Cyclin-dependent kinase (CDK) 4/6 activity, contributing to $G_{1}$ phase cell cycle arrest (28-30). The p21 mRNA level was decreased in A549/DDP cells depletion of BCLAF1 (Fig. 5C). Therefore, the cell cycle status of A549/WT and A549/DDP cells was subsequently characterized. A549/DDP cells compared with A549/WT cells were more arrested at the $\mathrm{G}_{1}$ phase (Fig. 5D). To assess if BCLAF1 may also serve a role in regulating cell cycle progression in A549/DDP cells, the effect of BCLAF1-knockdown on cell cycle progression was also examined. Notably, depletion of
BCLAF expression was demonstrated to reduce $\mathrm{G}_{1}$ arrest by 0.86 -fold in A549/DDP cells (Fig. 5D). These results suggested that BCLAF1 can induce $\mathrm{G}_{1}$ phase cell cycle arrest by regulating $\mathrm{p} 21$ and cyclin $\mathrm{D} 1$ expression.

\section{Discussion}

Lung cancer has one of the highest rates of incidence and is the leading cause of cancer-associated mortality worldwide (1). Although chemotherapy can be an effective therapeutic intervention strategy for the treatment of NSCLC, NSCLC gradually develops resistance to chemotherapeutic agents, such as cisplatin $(3,5)$; the underlying molecular mechanism of which remains poorly understood. In the present study, elevated BCLAF1 expression was observed in cisplatin-resistant A549/DDP cells, whilst downregulation of BCLAF1 expression was found to reverse cisplatin resistance in A549/DDP cells, suggesting that BCLAF1 serves an important role in the regulation of cisplatin resistance in NSCLC.

BCLAF1 has previously been reported to be involved in a number of biological processes $(12,13,31)$. It regulates gene transcription by mediating the formation of BRCA1-mRNA splicing complexes and modulating the DNA damage response by stabilizing the $\mathrm{Ku} 70 / \mathrm{DNA}$-dependent protein kinase complex during non-homologous end joining (NHEJ) (17). Since increased repair of drug-induced DNA damage and cell cycle alterations are two major mechanisms underlying chemotherapeutic resistance (32-34), the potential function of BCLAF1 on these two processes was investigated in A549/DDP cells in the present study. The key finding of the present study is that increased BCLAF1 expression in A549/DDP cells accelerated DNA damage repair and cell cycle progression. These observations suggest that BCLAF1 can induce cisplatin resistance in lung cancer cells by regulating the cell cycle and DNA damage repair.

It has been demonstrated that BCLAF1 interacts with $\gamma \mathrm{H} 2 \mathrm{AX}$ to stabilize the complex, promoting NHEJ-based DSB repair in cancer cells following irradiation $(14,16)$. A previous study revealed that BCLAF1 interacts with BRCA1 and thyroid hormone receptor associated protein 3, forming a BRCA1-interacting RNA splicing complex in response to DNA damage (17). This complex functions as a transcriptional regulator to selectively control the expression of a subset of genes associated with the DNA damage response, promoting efficient homologous recombination (HR)-mediated repair and repair of DNA interstrand crosslinks (14). These previous findings suggest that BCLAF1 serves a crucial role in the repair of double stranded breaks not only by NHEJ repair but also by HR repair. The current study further suggested elevated expression of BCLAF1 conferred cisplatin resistance in A549/DDP cells by increasing DNA repair capacity.

In addition to its role in the DNA damage response, BCLAF1 can also indirectly promote changes in cell cycle progression or the DNA damage response through transcriptional regulation (27). BCLAF1 is an essential component of a BRCA1-mRNA splicing complex $(14,17)$. In the present study, it was found that the loss of BCLAF1 expression in A549/DDP cells lead to reductions in USP22 and p21 expression, which partially supports the role of BCLAF1 in selective mRNA splicing and the export of mRNA encoding key DNA 
damage response proteins. Increasing BCLAF1 expression was revealed to contribute to cisplatin resistance by targeting p21 and cyclin D1 expression. p21 is a negative regulator of cyclin D-CDK4/6 activity and is sufficient to inhibit cell cycle progression during the $G_{1}$ and $S$ phases $(30,35,36)$. Elevated p21 expression protected A549/DDP cells from cisplatin-induced apoptosis by inhibiting DNA synthesis during $S$ phase, suggesting that one potentially important mechanism for acquired cisplatin resistance is that high expression levels of BCLAF1 can inhibit DNA synthesis through p21-induced $\mathrm{G}_{1}$ arrest. The present study had limitations. No clinical samples and clinically relevant conditions were investigated hence clinical relevance was not assessed. The findings of the present study indicate that BCLAF1 is likely a novel target mediating cisplatin resistance. Future studies targeting BCLAF1 in therapeutic practice are required to determine the true role of BCLAF1 in tumor suppression.

\section{Acknowledgements}

Not applicable.

\section{Funding}

This work was supported by grants from National Natural Science Foundation of China (grant nos. 81572775 and 81773004 to FZ) and the Program for Professor of Special Appointment (Eastern Scholar) at Shanghai Institutions of Higher Learning (grant no. TP2014055 to FZ).

\section{Availability of data and materials}

The datasets used and/or analyzed during the current study are available from the corresponding author on reasonable request.

\section{Authors' contributions}

TJ and BL performed the experiments, analyzed the data, created the figures and wrote the manuscript. FZ and DW conceived the project, designed the experiments and revised the manuscript. All authors read and approved the final manuscript.

\section{Ethics approval and consent to participate}

Not applicable.

\section{Patient consent for publication}

Not applicable.

\section{Competing interests}

The authors declare that they have no competing interests.

\section{References}

1. Wang J, Zhang ZQ, Li FQ, Chen JN, Gong X, Cao BB and Wang W: Triptolide interrupts rRNA synthesis and induces the RPL23MDM2p53 pathway to repress lung cancer cells. Oncol Rep 43: 1863-1874, 2020.
2. Bray F, Ferlay J, Soerjomataram I, Siegel RL, Torre LA and Jemal A: Global cancer statistics 2018: GLOBOCAN estimates of incidence and mortality worldwide for 36 cancers in 185 countries. CA Cancer J Clin 68: 394-424, 2018.

3. Morgensztern D, Campo MJ, Dahlberg SE, Doebele RC, Garon E, Gerber DE, Goldberg SB, Hammerman PS, Heist RS, Hensing T, et al: Molecularly targeted therapies in non-small-cell lung cancer annual update 2014. J Thorac Oncol 10 (Suppl 1): S1-S63, 2015

4. Janssen-Heijnen ML, van Erning FN, De Ruysscher DK, Coebergh JW and Groen HJ: Variation in causes of death in patients with non-small cell lung cancer according to stage and time since diagnosis. Ann Oncol 26: 902-907, 2015.

5. Zappa C and Mousa SA: Non-small cell lung cancer: Current treatment and future advances. Transl Lung Cancer Res 5: 288-300, 2016.

6. Fennell DA, Summers Y, Cadranel J, Benepal T, Christoph DC, Lal R, Das M, Maxwell F, Visseren-Grul C and Ferry D: Cisplatin in the modern era: The backbone of first-line chemotherapy for non-small cell lung cancer. Cancer Treat Rev 44: 42-50, 2016.

7. Perse $\mathrm{M}$ and Večerić-Haler Z: Cisplatin-induced rodent model of kidney injury: Characteristics and challenges. Biomed Res Int 2018: 1462802, 2018.

8. Dasari S and Tchounwou PB: Cisplatin in cancer therapy: Molecular mechanisms of action. Eur J Pharmacol 740: 364-378, 2014.

9. Kartalou M and Essigmann JM: Mechanisms of resistance to cisplatin. Mutat Res 478: 23-43, 2001.

10. Rocha CRR, Silva MM, Quinet A, Cabral-Neto JB and Menck CFM: DNA repair pathways and cisplatin resistance: An intimate relationship. Clinics (Sao Paulo) 73 (Suppl 1): e478s, 2018.

11. Kasof GM, Goyal L and White E: Btf, a novel death-promoting transcriptional repressor that interacts with Bcl-2-related proteins. Mol Cell Biol 19: 4390-4404, 1999.

12. Sarras H, Azami SA and McPherson JP: In search of a function for BCLAF1. ScientificWorldJournal 10: 1450-1461, 2010.

13. Wen Y, Zhou X, Lu M, He M, Tian Y, Liu L, Wang M, Tan W, Deng Y, Yang X, et al: Bclaf1 promotes angiogenesis by regulating HIF-1 $\alpha$ transcription in hepatocellular carcinoma. Oncogene 38: 1845-1859, 2019.

14. Vohhodina J, Barros EM, Savage AL, Liberante FG, Manti L, Bankhead P, Cosgrove N, Madden AF, Harkin DP and Savage KI: The RNA processing factors THRAP3 and BCLAF1 promote the DNA damage response through selective mRNA splicing and nuclear export. Nucleic Acids Res 45: 12816-12833, 2017.

15. Li X, He Z, Cheng B, Fang Q, Ma D, Lu T, Wei D, Kuang X, Tang S, Xiong $\mathrm{J}$ and Wang J: Effect of BCLAF1 on HDAC inhibitor LMK-235-mediated apoptosis of diffuse large B cell lymphoma cells and its mechanism. Cancer Biol Ther 19: 825-834, 2018.

16. Lee YY, Yu YB, Gunawardena HP, Xie L and Chen X: BCLAF1 is a radiation-induced $\mathrm{H} 2 \mathrm{AX}$-interacting partner involved in $\gamma \mathrm{H} 2 \mathrm{AX}$-mediated regulation of apoptosis and DNA repair. Cell Death Dis 3: e359, 2012.

17. Savage KI, Gorski JJ, Barros EM, Irwin GW, Manti L, Powell AJ, Pellagatti A, Lukashchuk N, McCance DJ, McCluggage WG, et al: Identification of a BRCA1-mRNA splicing complex required for efficient DNA repair and maintenance of genomic stability. Mol Cell 54: 445-459, 2014.

18. Carmody SR and Wente SR: mRNA nuclear export at a glance. J Cell Sci 122: 1933-1937, 2009.

19. Zhang F, Wu J and Yu X: Integrator3, a partner of single-stranded DNA-binding protein 1, participates in the DNA damage response. J Biol Chem 284: 30408-30415, 2009.

20. Livak KJ and Schmittgen TD: Analysis of relative gene expression data using real-time quantitative PCR and the 2(-Delta Delta C(T)) method. Methods 25: 402-408, 2001.

21. Galluzzi L, Senovilla L, Vitale I, Michels J, Martins I, Kepp O, Castedo M and Kroemer G: Molecular mechanisms of cisplatin resistance. Oncogene 31: 1869-1883, 2012.

22. Chen P, Li J, Chen YC, Qian H, Chen YJ, Su JY, Wu M and Lan T: The functional status of DNA repair pathways determines the sensitization effect to cisplatin in non-small cell lung cancer cells. Cell Oncol (Dordr) 39: 511-522, 2016.

23. Qin C, Zhang R, Lang Y, Shao A, Xu A, Feng W, Han J, Wang M, $\mathrm{He} \mathrm{W}, \mathrm{Yu} \mathrm{C}$ and Tang J: Bclaf1 critically regulates the type I interferon response and is degraded by alphaherpesvirus US3. PLoS Pathog 15: e1007559, 2019. 
24. Kinner A, Wu W, Staudt C and Iliakis G: Gamma-H2AX in recognition and signaling of DNA double-strand breaks in the context of chromatin. Nucleic Acids Res 36: 5678-5694, 2008.

25. Fragkos M, Jurvansuu $J$ and Beard P: H2AX is required for cell cycle arrest via the p53/p21 pathway. Mol Cell Biol 29: 2828-2840, 2009.

26. Wang A, Ning Z, Lu C, Gao W, Liang J, Yan Q, Tan G and Liu J: USP22 induces cisplatin resistance in lung adenocarcinoma by regulating $\gamma \mathrm{H} 2 \mathrm{AX}$-mediated DNA damage repair and Ku70/Bax-mediated apoptosis. Front Pharmacol 8: 274, 2017.

27. Meng X, Yang S and Camp VJA: The interplay between the DNA damage response, RNA processing and extracellular vesicles. Front Oncol 9: 1538, 2020.

28. Huang Y, Liu C, Zeng WC, Xu GY, Wu JM, Li ZW, Huang XY, Lin RJ and Shi X: Isoliquiritigenin inhibits the proliferation, migration and metastasis of Hep3B cells via suppressing cyclin D1 and PI3K/AKT pathway. Biosci Rep 40: BSR20192727, 2020.

29. Chen S, Zhou Q, Guo Z, Wang Y, Wang L, Liu X, Lu M, Ju L, Xiao Y and Wang X: Inhibition of MELK produces potential anti-tumour effects in bladder cancer by inducing G1/S cell cycle arrest via the ATM/CHK2/p53 pathway. J Cell Mol Med 24: 1804-1821, 2020.

30. Kreis NN, Louwen F and Yuan J: The multifaceted p21 (Cip1/Waf1/CDKN1A) in cell differentiation, migration and cancer therapy. Cancers (Basel) 11: 1220, 2019.
31. Dell'Aversana C, Giorgio C, D'Amato L, Lania G, Matarese F, Saeed S, Costanzo AD, Petrizzi VB, Ingenito C, Martens JHA, et al: miR-194-5p/BCLAF1 deregulation in AML tumorigenesis. Leukemia 31: 2315-2325, 2017.

32. Li HL, Wang CY, Fu J, Yang XJ, Sun Y, Shao YH, Zhang LH, Yang XM, Zhang XL and Lin J: PTEN expression in U251 glioma cells enhances their sensitivity to ionizing radiation by suppressing DNA repair capacity. Eur Rev Med Pharmacol Sci 23: 10453-10458, 2019.

33. Kamarudin MNA, Sarker MMR, Zhou JR and Parhar I: Metformin in colorectal cancer: Molecular mechanism, preclinical and clinical aspects. J Exp Clin Cancer Res 38: 491, 2019.

34. Yilmaz TE, Taşdemir M, Kaya M, Arican N and Ahishali B: The effects of magnesium sulfate on cyclophosphamide-induced ovarian damage: Folliculogenesis. Acta Histochem 122: 151470, 2020.

35. Warfel NA and El-Deiry WS: p21WAF1 and tumourigenesis: 20 years after. Curr Opin Oncol 25: 52-58, 2013.

36. Abbas T and Dutta A: p21 in cancer: Intricate networks and multiple activities. Nat Rev Cancer 9: 400-414, 2009.

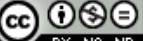

This work is licensed under a Creative Commons Attribution-NonCommercial-NoDerivatives 4.0 International (CC BY-NC-ND 4.0) License. 